\title{
Tiempo de excepción: El extraño retorno de la religión en política
}

\author{
Time of exception: The strange return of religion to politics \\ ÓSCAR GUARDIOLA-RIVERA \\ Universidad de Londres, Birkbeck College
}

Resumen. Bien se sabe que el alemán Carl Schmitt interpretó la posición del soberano en términos teológico-políticos: como el príncipe medieval cristiano cuya función consistía en prevenir y deferir el fin. Apuntalando las cercanías entre Schmitt y su mentor intelectual Thomas Hobbes, puede verse que en últimas la posición del decisor soberano es aquí la de quien intenta administrar o controlar el riesgo de la ocurrencia de eventos catastrófi$\cos$ en el futuro. Se argumenta en este ensayo que dicha forma de relacionarse con la temporalidad y el infinito - apocalíptica, autoritativa, y catastrofista - caracteriza tanto a la estética como a la economía y la política de autoridad que predominan en nuestra época. Se trata de un inesperado retorno de lo religioso en el corazón mismo de la modernidad, que anima la radicalización decisionista de los discursos político y económico, y sus presentaciones en la cultura popular tras la introducción de la guerra en el centro de la promesa moderna del progreso.

Palabras clave: Estado de excepción, religión, teología política, catástrofe, tiempo, infinitud.

\section{La guerra es padre de todas las cosas}

El punto de partida de este ensayo consiste en tomarse en serio la radicalización de
ABstract. It is well known that the controversial German theorist Carl Schmitt understood the position of the Sovereign in theologico-political terms: as similar to that of the medieval Prince, charged with the task of preventing and deferring the end of days. An exploration of the links between Schmitt and his acknowledged intellectual mentor Thomas Hobbes reveals that, ultimately, what is at stake in such understandings of the sovereign's position is the management and control of the risk associated with the possible occurrence of catastrophic events in the future. It is argued here that such a conception of our relationship with time and infinitude has come to dominate the political, economic and even aesthetic landscape of our times. That conception is apocalyptic, authority-based, and catastrophic; it amounts to an unexpected return of religion at the very heart of modernity, on the back of seemingly secular appeals to choice and necessity, following the introduction of war at the centre of the promise of progress.

Key words: State of exception, political theology, catastrophe, time, infinitude.

los discursos político, geopolítico, jurídico, y económico que ha tenido lugar tras la introducción de la guerra y el conflicto en el corazón de la promesa moderna del progreso y el desarrollo. En tal sentido 
debe entenderse la siguiente afirmación: el manto de benevolencia con el cual suelen arroparse los discursos del desarrollo y el progreso, con su invocación constante de los derechos, la libertad, y el pueblo «disfraza una terca voluntad de administrar y contener el desorden antes que resolverlo (...) Dado el constante hacer y rehacer de las sociedades que demanda el imperativo del progreso, el desarrollo se ocupa en forma particular de aquellos grupos y comunidades que, debido a contingencias tales como la miseria, el género o la falta de voz, quedan expuestas de manera regular a una situación de redundancia, convirtiéndose de tal manera en poblaciones superfluas incapaces de acceder a los requerimientos y recursos necesarios para vivir una vida aceptable» (Duffield, 2007: viii).

El punto - sugerido ya en los trabajos de Michel Foucault (1994: 367-382; 2002: 365-381; 2007)_ es que tales poblaciones superfluas, un verdadero resto de las operaciones del progreso, quedan expuestas al riesgo o a convertirse en riesgo ellas mismas. El desarrollo aparece en este punto como una tecnología política de seguridad a escala planetaria que a través del apadrinamiento y la tutela moral y educativa forma a las masas restantes de manera cuidadosa y vigilante en las artes de la libertad y la autogestión con el fin de hacerlas «útiles, incluidas y gobernables» (ibid.).

Aunque se trata de quienes no tienen lugar ni parte en el orden de cosas, el tutelaje desarrollista las incluye en el orden establecido - como un pueblo cuya unidad se exacerba ante la presencia de un obstáculo externo, que las más de las veces toma la forma del enemigo exterior o interno, el extranjero o el apátrida - conciliando de tal manera las demandas en principio incompatibles de orden con los azares del progreso. En este sentido, como afirma Mark Duffield haciendo eco de los trabajos del antropólogo Latinoamericano Arturo Escobar (1995), el desarrollo provee una respuesta liberal a las prácticas de exterminio y eugenesia, las otras «soluciones» que la modernidad ha puesto en práctica a la hora de enfrentar el problema de las poblaciones sobrantes y la competencia geopolítica por espacio vital o nuevo (Arrighi, 1994; 2007: 211249), así esté definido de manera constante por su relación con ellas. Lo anterior indica además que el desarrollo como tecnología política de seguridad, siempre relativa a un estado de emergencia o excepción (Duffield, 2007; viiixix), tiene origen o al menos está conectado en términos genealógicos con las estrategias de gobierno propias de la colonialidad.

Resulta importante entonces, establecer el tipo de relación que exista entre la colonialidad como estrategia de poder (Quijano, 2000: 201ss.) y el estado de emergencia o excepción normalizada en el que resultan siempre las políticas de autoridad y seguridad. Se trata de una conexión que algunos analistas del recurso constitucional de excepción niegan o dejan de lado (Agamben, 1998) mientras otros enfatizan (Hussain, 2003, 15 ss.), con el resultado para unos de la conversión del estado de excepción en una categoría cuasi-existencial, à la Schmitt, mientras que para otros la normalización o difusión del estado de excepción durante y con posterioridad a la época de las guerras mundiales hace parte de las permutaciones del poder estatal cuando éste actúa más allá del dominio territorial establecido de su soberanía y entonces «materializa su soberanía como Imperio» (Butler y Spivak, 2007: 10; Hussain, 2003 y 2007: 734-753).

El controvertido constitucionalista alemán Carl Schmitt, - muy influyente entre las generaciones más y menos recientes de estudiantes de leyes, contem- 
poráneos y posteriores al ascenso del falangismo en la Península Ibérica, que en España, Argentina, Colombia y el resto de América Latina aprendieron sus primeras lecciones de política y derecho constitucional en las páginas de El Concepto de lo Político (1998) y los textos de constitucional del español Luis Legaz y Lacambra - transformó como bien se sabe la discusión sobre las condiciones excepcionales, hasta entonces signada por debates acerca de si la última palabra correspondía al Ejecutivo o al Legislativo, o acerca del criterio apropiado para establecer si existía o no estado de guerra, al hacer de la decisión sobre la existencia y manejo de tales condiciones, o su declaración por un soberano personalizado, la clave del concepto de Soberanía.

Al tiempo, como bien se sabe, Schmitt $(1985 ; 1996)$ interpretó la posición de tal soberano en términos teológico-políticos, a la manera del príncipe medieval cristiano cuya función consistía en prevenir y deferir la llegada del anticristo. Si se la separa un momento de sus connotaciones más teológicas, puede verse que en últimas la posición del decisor constitucional en la explicación que da Schmitt es la de aquel que intenta administrar o manejar la ocurrencia de eventos catastróficos en el futuro - finalmente el más catastrófico de todos, el fin de los tiempos-. Es esta forma de relacionarse con la temporalidad, la historia y el infinito lo que caracteriza a la política de autoridad que predomina en nuestra época. Dicha característica será el objeto más o menos explícito de las páginas que siguen.

Así pues, resulta crucial insistir en que lo propio de quien decide es la prefiguración especulativa y la prevención, la lectura del futuro dentro de un marco que ya ha totalizado la temporalidad al encerrarla en un esquema apocalíptico. Lo que lleva al decisor político a suspender y sustraer la legalidad y las regulaciones es
- como lo percibieron contradictores contemporáneos de Schmitt como los escritores judíos Jacob Taubes y Walter Benjamin (1980, 1: 245 ss.) — un profundo miedo que es al tiempo una profunda atracción por el fin y el más allá. La política de autoridad es entonces una política del fin y del miedo al fin. En otros términos, es una política del deseo de muerte.

En este punto cabe hacer al menos dos interpretaciones: primera, la política de autoridad es una política del miedo que esconde tras de sí un oscuro objeto de deseo. Se trata de una interpretación psicologizante, cercana a la teoría sicoanalítica, de acuerdo con la cual el afecto predominante en la actualidad, el resentimiento, es un afecto triste cuyo origen se encuentra en la manera persistente como percibimos el sufrimiento de otros «a distancia» - en particular a través de los medios electrónicos audiovisuales - sin que en verdad esa distancia se acorte, sin que el evento, cuya realidad no ignoramos, nos convierta en verdaderos militantes. Dicho de otra forma, el evento lo es para otros mas no para nosotros. Por ello, al tiempo que expresamos nuestra simpatía con las víctimas y nos lanzamos a la calle para vociferar en su nombre, en vez de ellas y no con ellas, expresamos también nuestro deseo de ocupar el lugar de la víctima y contener su afecto, y nuestra frustración al no poder hacerlo del todo; el deseo de experimentar el dolor real, el olor o el hedor de la jungla a la manera de quien se adentra en el corazón de las tinieblas. Nuestros actos son en este sentido «actos hacia fuera», golpes de pecho públicos, exagerados, masivos, y sin embargo nada más que formaciones de defensa. Desearíamos poder sentir el hedor, la ansiedad y hasta el dolor de la víctima, o gritar en medio del espanto, el pánico y el escándalo general como ella lo hace (en el espacio «exterior» en el cual, como en el filme Alien, nadie escucha su 
lamento), sin que llegásemos en verdad a ocupar su lugar o enfrentar su rostro ${ }^{1}$.

Quizás valga la pena en este sentido, prestar atención a la manera en que el terror apocalíptico ha penetrado el corazón de la cultura popular, desde la taquillera película de Hollywood Cloverfield producida por el guionista de la serie de televisión Lost, J. J. Abrams, con sus reminiscencias de 9/11 en perspectiva de primera persona del singular, pasando por las menos exitosas o conocidas como Southland Tales de Richard Kelly, las series de televisión Invasion, Jericho y $\mathrm{Su}$ pernatural (que se desarrollan, no por coincidencia, en el «bible belt» de los Estados Unidos), la coreana The Host, la colombiana Satanás, las menos sofisticadas como el horror gore de Saw o The Hills Have Eyes, hasta la literatura fatalista de Chuck Palahniuk, la serie novelada Left Behind, el documental que ganó el Oscar en 2007, y la historia debida a un novelista colombiano en la cual un desalmado ejército revolucionario desciende sobre Bogotá.

Lo que es común a estas expresiones diversas de la cultura popular es un peculiar sentido del fin que cabría llamar, guardadas las proporciones, pauliano. La referencia es por supuesto a la teología política que se asocia al nombre de Pablo de Tarso y a su doble estrategia de presentar el choque de la excepción en contraste con un fondo de continuidad y normalidad carentes de eventualidad. Aquí también, en nuestra atención y preferencia por este tipo de cultura y por esta estética, se expresan a una nuestra simpatía con el dolor de las víctimas y nuestro deseo de saborear la realidad impaladeable del mundo afuera de nuestras comunidades cerradas y nuestras ciudades pacificadas, siempre manteniendo una distancia razonable.

Una segunda interpretación pasa por reconocer que la política de autoridad triunfa porque politiza nuestra concepción del tiempo y de la historia al asociarse con la posibilidad de que exista, como construcción social, estética, y política, un estado o forma de vida más allá del espacio de la normalidad. Podríamos llamar a esta una voluntad de trascendencia, más paradójica cuanto más reconozcamos lo difícil que resulta hablar de trascendencia en una época secularizada (Weber, 2008: 184-9). Y cuando la normalidad se identifica con un estado de cosas indeseable, no debería resultar extraño el que la gente opte por una política de choque, que corresponda a y nos lleve más allá de la anormalidad de los tiempos, esto es, que realice nuestra voluntad de trascendencia. Más aún, dado que una situación de «normalidad» no puede ser interrumpida desde dentro, se concluye entonces que debe serlo desde afuera por una persona que declare su excepcionalidad y viniendo desde el exterior - trascendiendo a los ciudadanos y a la ley ciudadana que ella encarna - interrumpa de tal manera la línea de continuidad.

\section{El retorno inesperado de la teología política}

Desde esta perspectiva, como observó Schmitt (1985: 36), la teoría y la práctica política comienzan a aproximarse a la teología política. En esta aproximación lo que se demanda es que exista una suerte de Corte de Apelación localizada por fuera de todos los marcos establecidos que defina el carácter de los tiempos, o bien, lo produzca. Éste es el estado de excepción, que puede ser descrito como una cesación del tiempo ordinario (Bredekamp, 1999: 252). La disparidad entre la continuidad y la discontinuidad se extiende aquí hasta incluir una concepción de la temporalidad que distingue entre normalidad y excepción. Esta idea es quizás la clave del pensamiento constitucional 
schmittiano, expresado en el dictum según el cual «soberano es aquel que decide sobre el estado de excepción» («Souverän ist, wer über der Ausnahmezustand entscheidet») ${ }^{2}$. No puede sorprender que una idea tal seduzca a tirios y a troyanos, a hombres de izquierdas y derechas, a jóvenes conversos descontentos y a neoconservadores maduros, pues mezcla de una vez la definición universal y la fundamentación de lo que permanece con la idea de trascendencia más allá de la normalidad impotente y aburrida. Se trata de una concepción del tiempo seductora e inusual, que se hace aún más fascinante cuando se establece y hace concreta en términos de «prevención», «retraso»y «voluntad de deferir» o trascender.

La interpretación del estado de excepción como «retraso» (o «Frist», en el original alemán) debida a Schmitt es, como explica Horst Bredekamp, una función de su concepción del Príncipe y el Soberano como katechon o «Retenedor»; esto es, el que impide o retrasa la llegada del anticristo, en la clave apocalíptica y mesiánica expresada por Pablo en Tesalonicenses 6 y 7. En este esquema la historia es el espacio de tiempo que discurre entre el presente y la llegada del anticristo, cuando las acciones del Sumo Ilegal, el Malvado, no tienen las de ganar y el anticristo no ha aparecido aún entre nosotros. El papel del katechon es producir historia al retrasar o deferir el lapso de tiempo que llevaría a la contra-temporalidad de la llegada y aparición del anticristo, bien sea que este aparezca en la forma del comunismo o de la mecanización del mundo y el materialismo. Sin él, sin el katechon, el protector o decisor que retrasa la llegada del enemigo (el comunismo, la mecanización, el materialismo o cualquier otra forma de la catástrofe final) el tiempo habría llegado a su fin desde hace mucho. Como puede verse entonces, es en el juego recíproco entre la continuidad normal y el choque del estado de excepción que se genera historia; empero, y esto es lo más importante, tal historia aparece tan solo como Frist, como retraso y deferir, o como cabría decir en un sentido más secular y político, la voluntad de trascendencia se hace concreta como prevención.

Aunque parezca críptica al comienzo, no cabe dudar del poder seductor de esta idea pues supone la existencia de una zona más allá del mundo conocido en la cual las reglas de lo normal se anulan y tiene lugar un momento de claridad y abrupta interrupción semejantes a la experiencia de epifanía o conversión. A tal motivo corresponden también las ideas del choque, el ahora o el instante, y la inmanencia e inmediatez propias de la estética de vanguardia propagada por Aragon, Bataille y Breton. Así mismo la crítica de Walter Benjamin ${ }^{3}$, y en general la estética del shock (como por ejemplo en el militarismo estetizado del «shock and awe») que hoy predomina en nuestra cultura mediática visual. Y si a semejante politización de la temporalidad se une el poder espectacular de una estética del choque y la singularidad, deberá entenderse el poder y la fascinación que ejerce sobre unos y otros. En este punto convergen las estéticas surrealista y constructivista originadas en Europa a comienzos del siglo XX, predominantes en la cultura visual contemporánea ${ }^{4}$, y la visión paulina de la interrupción mesiánica. El corolario es el siguiente: si de un lado actuamos en el papel de la víctima, y lo hacemos de manera más o menos bien intencionada, guardando cierta distancia, del otro pretendemos abandonar la indolencia, la impotencia, y el aburrimiento de la situación presente al interrumpir, con un efecto de choque, la continuidad de la historia.

Se trata entonces de experimentar el placer unido al dolor pero sustrayendo el 
componente indeseable de este último, de testificar en nombre de las víctimas, en lugar de ellas, al declararlas símbolo universal de una violencia total, indecible, y de interrumpir la continuidad del paso del tiempo para dar lugar a un momento de claridad y revelación, experimentado en común, con el fin de prevenir el cierre o preclusión de los tiempos que significaría la llegada de una hecatombe final o su personificación en una figura catastrófica, contaminante y maligna.

Dado el carácter central que en este proceso adquieren la visualización, la imagen y la representación, el lector atento a la historia de las ideas políticas y constitucionales mal podría desatender la conexión entre esta versión más contemporánea de la teología política y la debida en época más temprana al escritor inglés Thomas Hobbes. En ambos casos se trata de épocas pos - o contrarrevolucionarias, cuyo enfoque se centra en el trauma individual o colectivo que dejan la rebelión y el recurso a la violencia, y más importante aún, en ambos casos las tecnologías visuales de proyección (hacia adelante en el tiempo y el espacio) reemplazan a los actores en el centro de la escena.

No en vano el propio Schmitt, cuyo intento por reactivar la visión hobbesiana del Estado como fundado en la muy elemental y humana aprehensión frente al miedo y el terror bien podría definir un elemento importante del imaginario político de nuestra época, reconoció en Hobbes a su mentor intelectual ${ }^{5}$. En efecto, la imagen que aparece en el frontispicio de su Leviatán ofrece una de las representaciones más efectivas de lo que el escritor del siglo XVII percibió como una experiencia colectiva de caos político y social en el período siguiente a la Revolución Inglesa. Como dice Bredekamp, cuya argumentación se ha seguido en los párrafos anteriores, esta representación "creada en el estilo arcimboldesco de las imágenes compuestas, con su cuerpo constituido por unas trescientas personas que, como una cota de malla, reemplazan la piel y de manera obvia se extienden para conformar el resto del cuerpo» produce un doble efecto «en el que cientos de personas tornan su mirada hacia una cabeza única que a su turno nos devuelve la mirada» presto a moverse hacia adelante, e ilustra el pasaje decisivo del capítulo 17 del Leviatán en el cual la «generación» del estado ocurre a través de la transferencia de las voluntades individuales al Soberano (Bredekamp, 1999: 257-8; Hobbes, 1991: 120 ss.). Bredekamp apunta de manera correcta que «generación» significa en este caso no sólo la creación de un cuerpo sino también el dar comienzo al tiempo, en consonancia con la doctrina medieval y la tradición quizás más antigua y presente en diversas culturas, de crear efigies tras la muerte del rey, o su equivalente, con el objeto de llenar el vacío que consume el período de interregno con una representación casi viva del Estado.

Se trata por supuesto de verdaderos fetiches, objetos cuya función es atrapar el tiempo, encadenarlo y permitir de tal manera la comunicación de los presentes (y el pasado) con el futuro. En tal sentido, estamos ante verdaderas tecnologías de temporalización y es precisamente en este sentido que las efigies estatuarias de reyes, faraones y emperadores preparan el camino para el proceso de visualización sin el cual el Leviatán sería inconcebible (Bredekamp, 1999: 258). El conjunto de efigies-fetiche hace visible lo que de otra manera solo sería perceptible al ojo supernatural que contempla el tiempo en su totalidad trascendente, esto es, como dice Bredekamp, «la cadena de quienes han ocupado el más alto oficio, fragmentada en el tiempo, y sin embargo extendiéndose desde el pasado hacia el 
futuro en una figura compuesta coherente» (íbid.). En efecto, Hobbes explora el llamado «derecho de sucesión» como si contemplase las efigies de los reyes desde el siglo catorce hasta el presente, y extendiéndose en el futuro ${ }^{6}$. Se trata de una tecnología de proyección que representa de manera efectiva las pretensiones del estado a la duración y la eternidad completas. En esta representación, el interregno es el centro alrededor del cual se mueven las consideraciones políticas; es el mismo lugar que ocupa en el esquema schmittiano el estado de excepción. Como puede verse, no se trata tan sólo de un medio de suspensión de la ley para confrontar circunstancias excepcionales, sino de una finalidad o su representación, o dicho de una vez, de una imagen del fin frente a la cual se alza, como un fin, la permanencia del Estado.

Ahora bien, mientras que a Hobbes preocupa el transformar la temporalidad potencialmente indefinible de la anarquía en un período de orden a través de la permanencia del contrato social y la efigie viviente, Schmitt concentra su atención en la duración de un orden inestable al cual él opone el estado de excepción. Para ambos el tema es la permanencia del Estado, llegado al ser tras un período «de naturaleza», y por lo tanto antinatural, pero que una vez llegado al ser aspira a la eternidad. Empero, si para Hobbes el punto es la permanencia del Leviatán, el Estado, Schmitt se ocupa en cambio de adicionar al componente visual y tecnológico un elemento afectivo que explicita el sentido de esta permanencia como un fin: la consecuencia del arreglo visual-tecnológico es afectiva, una forma de exaltación momentánea, similar a un éxtasis, o al pánico, que produce en los individuos el afecto de fidelidad $y$ casi-militancia que sigue a la epifanía y la conversión. Como sugiere Bredekamp, Schmitt, y otro tanto cabe decir de sus se- guidores, es un romántico de la ocasión excepcional.

Este conjunto de observaciones es de suma importancia: en primer lugar, nos sugiere que la política tiene como objetivo enfrentar la incertidumbre propia de la temporalidad, y en particular la temporalidad futura. En segundo lugar, aparece claro también desde esta perspectiva que la forma de enfrentar la incertidumbre consiste en prefigurar el futuro e imaginar, desde ese punto de vista, una temporalidad completa que incluye la cadena de interrupciones. En tercer lugar, se trata de mantenerse fieles a esa interrupción y militar a favor de ella extendiéndola hasta el infinito, universalizándola. Pero ¿cómo tiene lugar esta extensión? De una parte, hay que mantener abierto el período de la interrupción hasta el infinito, esto es, deferir su cierre. De otra parte, hay que concebir la temporalidad completa como un universo regular dentro del cual la interrupción se abre en una serie de posibilidades que pueden o no realizarse, como las ramas de un árbol o un desvío en el camino, todas ellas visibles al fiel observador.

En cuarto lugar, mantenerse fieles a la extensión universal del interregno implica en este caso postular la temporalidad completa como la forma de la necesidad por antonomasia, o dicho de otra manera, como una ley determinista o aleatoria que se extiende en el futuro prospectivo. Nótese cómo el supuesto que opera en esta postulación es que el cambio o la transformación radical debe ser infrecuente en el futuro, pues de otra manera la frecuencia del cambio haría el universo (la sucesión, la cadena de sucesos) inestable e inobservable. En quinto lugar, la imagen que interrumpe el flujo normal del tiempo y encadena y estabiliza la anormalidad, y abre por tanto la temporalidad a sus «infinitas» posibilidades, no puede ser abstracta y por ello mis- 
mo se la debe hacer concreta en objetos que son verdaderas tecnologías; por ejemplo una cadena estatuaria que extiende el pasado en el futuro, una efigie viviente presta a moverse hacia adelante, o un autómata. En este punto se encuentran las estrategias de una soberanía secularizada que aún tiende a la trascendencia, con los regímenes de verdad que se desarrollan a partir de los paradigmas cibernético y probabilístico. Un corolario importante es que, como bien se sabe, un autómata (también en cibernética) se regula a sí mismo. Otra consideración de importancia, una que pasa desapercibida a Hobbes, a Schmitt y a buena parte de quienes les siguen, es que el comportamiento de un autómata nunca es simple sino complejo.

A partir de esta última consideración sería posible desarrollar un argumento de acuerdo con el cual el reconocimiento de una forma específica de complejidad trae consigo un tipo de incertidumbre nueva y más radical, una que escapa a las estrategias de la soberanía secularizada trascendente aunque esté relacionada con ellas. Se trataría de una incertidumbre objetiva que escapa tanto a las distinciones de los principios de precaución y prevención, esta última entendida como observación fiel de la temporalidad abierta y completa, como a las operaciones de cálculo propias de la extensión de la estabilidad observable en un futuro prefigurado (la probabilística).

Para concluir: se ha sugerido en los párrafos anteriores que Hobbes y Schmitt descubren una forma eficaz y efectista de enfrentar la incertidumbre. Ésta consiste en la visualización de una cadena de decisiones coherente, tomadas por un agente supuestamente capaz de observar la totalidad del tiempo, y de encadenar y estabilizar la anormalidad, que cumpliría de tal manera con la pretensión de reducir la complejidad o trascenderla. Dicha «for- ma», eficaz y efectista, es el Estado (más tarde también el mercado) concebido(s) como un sistema autorregulado y capaz de enfrentar el riesgo.

Este último, el riesgo, es presentado a su vez como un estado de nuestra naturaleza: «miedo» en Hobbes, «indecisión» o «indecibilidad» en Schmitt, «terror» en el discurso populista de nuestros políticos neoconservadores y posrradicales. Se supone que tal estado se debe a los límites de nuestro conocimiento, presionado por una institucionalidad que hace de la información un recurso escaso pero en principio susceptible de corrección a través de más simples y mejores tecnologías e instituciones. Dicho mejoramiento y simpleza serían apreciables en la evolución que va de la concreción tecnológica del «derecho de sucesión» en una cadena de efigies estatuarias hasta el autómata de la gobernancia ejecutiva e imperativa de múltiples niveles, característica de los regímenes posdemocráticos o "globalizados» de hoy, tales como la Unión Europea, el fallido «Proyecto para un nuevo siglo americano» del Partido Republicano de los Estados Unidos o, guardadas las proporciones, la «Seguridad Democrática» de ciertos gobiernos latinoamericanos. En suma, se trata de en todos estos casos de reafirmar el principio de soberanía de acuerdo con el cual «el Uno equivale a los muchos». Estamos enfrente de una falsa trascendencia.

\section{El Estado contra la sociedad}

Es notable el que dicho autómata aparezca siempre en contraste con el fantasma de una sociedad sin poder trascendente que la organice, sin aparato de Estado que se oponga a la vida del común, una en la cual los muchos no pueden equivaler a la Unidad. Se trata de una confirmación, desde la orilla opuesta, de aquello que observaron los cronistas hispanos de los siglos XVI y XVII al describir las comunidades preco- 
lombinas de las Américas: que se trataba de organizaciones políticas fundadas sobre tecnologías de contrapoder, carentes de la figura del uno exterior a la sociedad, «pueblos sin fe, sin ley, sin rey» ${ }^{7}$. El término utilizado por cronistas como Pedro Cieza de León para designar tales comunidades era «behetrías», literalmente «pueblos sin cabeza». Esta literatura descriptiva produjo un efecto considerable en las mentes europeas, que de pronto despertaron a la pregunta propuesta por el estudiante de Leyes Etienne de la Boétie en la Francia de 1548: ¿Cómo es que hemos venido a desear la servidumbre y a defenderla, con nuestra sangre si ello llega a ser necesario?

En realidad se trata no de una sino de al menos tres preguntas: Primero: ¿Puede decirse que sigue siendo hombre aquel que en contra de su naturaleza ha decidido no ser más un hombre, es decir, un ser libre? Para Boétie, la existencia de sociedades sin estado en el Nuevo Mundo prueba que la obediencia es un estado histórico, un infortunio, y por lo tanto antinatural en el sentido en que se trata de una forma de obediencia muy diferente de la de los animales. Los hombres obedecen no por miedo a la muerte (como sugeriría Hobbes) sino que lo hacen de manera voluntaria, porque quieren, porque lo desean. Segundo: ¿Es el deseo de sumisión innato o adquirido? Tercero: ¿Cómo funcionan las sociedades «primitivas» a la hora de prevenir la aparición de un punto de acumulación de fuerza y poder, y entonces la desigualdad, la división jerárquica y las relaciones de poder? Esta última cuestión no la formula el autor del Discours de la servitude volontaire ${ }^{8}$ sino los etnólogos contemporáneos que desde hace ya varias décadas cuentan con una sobreabundancia de muestras etnográficas acerca de la operación de las sociedades mal llamadas primitivas.

Dicha literatura etnográfica se concentra en la manera como tales socieda- des controlan, codifican, y ritualizan la violencia al punto de reducirla si es que no abolirla. Con todo, también aquí se evoca la violencia con el fin de prevenirla, para demostrar el horror que ella causa en tales sociedades y mostrar a éstas como, finalmente, sociedades contrarias a la violencia. Por lo mismo no sorprende que en la etnología brille por su ausencia una reflexión sostenida acerca de la forma más aguda de violencia: la guerra. El lector de tales literaturas pensaría que la guerra no pertenece al funcionamiento normal de tales sociedades, que se trata de la excepción. Etnólogos más contemporáneos, Napoleón Chagnon y Pierre Clastres entre otros, dedicaron sus esfuerzos a mostrar cómo la exclusión de la guerra del discurso científico de la etnología no correspondía a la realidad descrita por el archivo iniciado por los cronistas de Indias. En el archivo, la agresividad de las poblaciones sin fe, derecho o monarca es central, se la juzga y percibe, quizás de manera perversa, como la causa de su desaparición. Con todo, es la aparente prevalencia de la guerra en la vida primitiva lo que cautiva la atención de estos primeros científicos sociales europeos. Thomas Hobbes por ejemplo, derivó como bien se sabe de tal manera su distinción entre el estado civil o de sociedad y el estado de naturaleza, descrito como la guerra de todos contra todos que persiste de manera concreta y observable «entre los pueblos salvajes de muchos lugares de América, en los cuales excepto por el gobierno de la familia, cuya concordia depende del deseo natural, no existe gobierno alguno; y tales pueblos viven de esta forma brutal, como dije antes» (Hobbes, 1991: 88).

Así pues que el «tiempo de anarquía» potencialmente infinito al que Hobbes opone, para transformarlo en orden y autoridad, el momento del interregno y la extendida excepción mediante la perma- 
nencia del contrato social y la efigie viviente no resulta ser otro que el tiempo antiestatal de las comunidades indígenas de las Américas. La dimensión colonial emerge en este punto, una dimensión de gobierno global que no pasa desapercibida al Carl Schmitt del Nomos de la Tierra en el Derecho Público Europeo. Ahora bien, para entender esta dimensión resulta importante preguntarse primero: ¿Cuál es el tiempo de tales comunidades? Responder a tal pregunta con suficiencia nos llevaría más allá de los límites de este trabajo, sin embargo bástenos con sugerir por lo pronto que se trata de una concepción del tiempo que podría llamarse «tiempo proyectado», una predicción informada e imaginada a la manera de un ciclo o un círculo incompleto, una espiral si se quiere, por oposición al «tiempo ocurrente» o trascendente que en la reflexión que va de Hobbes a Schmitt incluye en una totalidad completa la linealidad de la normalidad en juego recíproco con la excepción extática, es decir, el encadenamiento de la anormalidad y su extensión. Si en el segundo podemos continuar usando la imagen del árbol decisional, la línea que se bifurca, la decisión de la choice theory, en el primer caso habremos de utilizar la imagen del círculo o la espiral. Empero, como se sugirió antes, el desarrollar las implicaciones de esta imagen nos llevaría más allá de los límites de este ensayo. Deberemos entonces concluir en este punto, en cliffhanger, como dirían los escritores de ese subconsciente colectivo de nuestros días al que solemos referirnos con el nombre Hollywood.

\section{BIBLIOGRAFÍA}

Agamben, G. (2002): «La Inmanencia Absoluta», en Gilles Deleuze: Una Vida Filosófica. e-Book editado bajo la dirección de Eric Alliez. Traducido por Ernesto Hernández B. Cali y Medellín: Sé Cauto/Euphorion.

- (2000): Le temps qui reste. Paris: Editions Payot \& Rivages.

- (1998): Homo Sacer. Sovereign Power and Bare Life. Translated by Daniel Heller-Rozen. Stanford: Stanford University Press.

Arrighi, G. (2007): Adam Smith in Beijing: Lineages of the Twenty-First Century. Nueva York y Londres: Verso.

- (1994): The Long Twentieth Century: Money, Power, and the Origins of our Times. Nueva York y Londres: Verso.

BredeKAMP, H. (1999): «From Walter Benjamin to Carl Schmitt, via Thomas Hobbes», en Critical Inquiry, invierno 1999, pp. 247-266. Chicago: The University of Chicago Press.

Butler, J. \& SpivaK, G. C. (2007): Who Sings the Nation-State? Language, Politics, Belonging. Londres: Seagull Books.

Clastres, P. (1994): The Archeology of Violence. Nueva York: Semiotext(e).

De la Boétie, E. (1976): La Boétie: Le Discours de la servitude volontaire. Paris: Payot.

Douzinas, C. (2008): El Fin de los Derechos Humanos. Traducido al castellano por Óscar Guardiola-Rivera y Ricardo Sanín. Bogotá y Miami: Legis.

DufFIELD, M. (2001): Global Governance and the New Wars. Londres y Nueva York: Zed Books.

- (2007): Development, Security and Unending War. Governing the World of Peoples. Londres: Polity.

Foucault, M. (1994): Dits et écrits, 1954-1988, IV. Ed. Por D. Defert y F. Ewald. Paris: Éditions Gallimard.

- (2002): Power. Essential works of Foucault, 1954-1984, volume 3. ed. Por James D. Faubion. Londres: Penguin. 
- (2007): Security, Territory, Population. Lectures at the College de France, 1977-1978. Editado por M. Senellart. Traducido por G. Burchell. Basingstoke: Palgrave MacMillan.

Hussain, N. (2003): The Jurisprudence of Emergency: Colonialism and the Rule of Law. Ann Arbor: University of Michigan.

- (2007): «Beyond Norm and Exception: Guantánamo», en Critical Inquiry, vol. 33, n. 4, verano 2007, pp. 734-753. Chicago: The University of Chicago Press.

QuiJano, A. (2003): «Colonialidad del Poder, Eurocentrismo y América Latina», en La Colonialidad del Saber: Eurocentrismo y Ciencias Sociales. Perspectivas Latinoamericanas. Buenos Aires: CLACSO.

Schmitt, C. (1985): Politische Theologie, Vier Capitel zur Lehre von der Souveränität. Berlin: Duncker \& Humboldt.
- (2005): Political Theology: Four Chapters on the Concept of Sovereignty, trad. George Schwab. Chicago: Chicago University Press.

- (1996): [1932] The Concept of the Political. Trad. Por G. Schwab. Chicago: Chicago University Press.

- (1998): El Concepto de lo Político. Madrid: Alianza Editorial.

- (1938): Der Leviathan in der Staatslehre des Thomas Hobbes: Sinn und fehlschlag eines politischen Symbols, editado por G. Maschke, Colonia.

- (1936): «Der Staat als Mechanismus bei Hobbes und Descartes», en el Archiv für Rechts- und Sozialphilosophie 30, pp. 622-32.

TAussig, M. (2008): «Zoology, Magic, and Surrealism in the war on Terror», en Critical Inquiry, vol. 34, n. 5, pp. s98-s116. Chicago: Chicago University Press.

WeBer, S. (2008): Benjamin's - abilities. Cambridge, Ma.: Harvard University Press.
1 Sobre el significado de enfrentar el rostro de la víctima o «el otro» en el discurso y la práctica de los derechos humanos, véase Douzinas, C. (2008) El Fin de los Derechos Humanos. Bogotá y Miami: Legis, capítulo 13.

2 Véase Schmitt (1985: 11) y también (2005: 13).

3 Para la relación entre Benjamin y Schmitt véanse Bredekamp (1999) y Weber (2008: 176-194).

${ }^{4}$ Sobre este particular véase Taussig (2008: s98-s116).

5 Véanse por ejemplo sus escritos (1936) «Der Staat als Mechanismus bei Hobbes und Descartes» en el Archiv für Rechts- und Sozialphilosophie 30, pp. 622-32 y (1938) Der Leviathan in der Staatslehre des Thomas Hobbes: Sinn und fehlschlag eines politischen Symbols, editado por G. Maschke, Colonia.

6 Véase Hobbes (1991) Leviathan, p. 135.

${ }^{7}$ Pierre Clastres citando a las cronistas españoles del siglo XVI. Véase (1994) The Archeology of Violence. Nueva York: Semiotext(e), p. 140.

8 De la Boétie, E. (1976) La Boétie: Le Discours de la servitude volontaire. Paris: Payot. 